\title{
Putting muscle in DNA methylation
}

\author{
James P Reddington ${ }^{1}$, Richard R Meehan ${ }^{1,2}$ \\ ${ }^{I}$ MRC Human Genetics Unit, IGMM, Western General Hospital, Edinburgh EH4 2XU, UK, ${ }^{2}$ Breakthrough Research Unit, Univer- \\ sity of Edinburgh, Western General Hospital, Edinburgh EH4 2XU, UK \\ Cell Research (2011) 21:1531-1533. doi:10.1038/cr.2011.91; published online 31 May 2011
}

Over 25 years ago seminal experiments from the labs of Peter Jones and Harold Weintraub demonstrated that alteration in the DNA modification state underlie the myogenic conversion of fibroblast cell lines [1, 2]. This paved the way for the identification of myogenic helix-loop-helix (HLH) proteins in muscle differentiation, but the mechanism by which DNA methylation regulates muscle differentiation has remained elusive [3]. In general, DNA methylation at the 5'-position of cytosine (in $\mathrm{CpG}$ dinucleotides) is associated with transcriptional repression and implicated in maintaining patterns of gene expression between different cell types and during differentiation. How DNA methylation contributes to a gene regulatory network that directs spatial, temporal and ultimately lineage restricted patterns of gene expression in the developing embryo is still unclear. One proposed mechanism involves the binding of methyl-CpG-binding proteins (MeCPs) to methylated DNA that can be viewed as 'reading the mark'. Various protein domains have an affinity for methylated cytosine including the methyl-binding domain (MBD) of $\mathrm{MeCP} 2$ and the zinc-finger domains of the Kaiso-like protein family [4].

Correspondence: Richard R Meehan ${ }^{\mathrm{a}}$, James P Reddington ${ }^{\mathrm{b}}$

aE-mail: Richard.Meehan@hgu.mrc.ac.uk

bE-mail: james.reddington@hgu.mrc.ac.uk
MeCPs associate with heterochromatin in nuclei and recruit silencing complexes to reduce the transcription potential of methylated genes.

Genetic analysis of the four MBDcontaining proteins in mammals capable of binding methylated DNA: MeCP2, MBD1, MBD2 and MBD4, has not implicated them in muscle development [4]. A second class of proteins, the Kaiso-like family of methyl-CpGbinding proteins (Kaiso (ZBTB33), ZBTB4 and CIBZ (ZBTB38)), have also been associated with transcriptional repression of methylated reporter genes $[5,6]$. However, in general, mice lacking MeCPs display very minor or no developmental phenotypes [4], whereas mice lacking DNA methyltransferases, Dnmt1 or Dnmt3b, show severe developmental defects including reduced somite formation in the case of Dnmt1 [7, 8]. This contrasts with Xenopus laevis, where $\mathrm{xKaiso}$ and $\mathrm{xMeCP} 2$ appear to have a more prominent role in embryonic development. Depletion of $\mathrm{xMeCP} 2$ in Xenopus embryos leads to altered neural patterning and embryonic lethality through mis-expression of another HLH factor, $x$ Hairy $2 a$ [9] and depletion of xKaiso leads to premature activation of zygotic transcription and embryonic lethality [10].

Recently, Oikawa et al. [11] advanced our understanding of a less wellstudied methyl-CpG-binding protein, CIBZ, by studying its role in muscle differentiation through regulation of
Myogenin, a gene encoding a basic HLH protein that is an essential musclespecific transcription factor [12]. CIBZ (CtBP-interacting BTB zinc finger protein, ZBTB38) possesses a modified zinc finger domain capable of binding methylated DNA in vitro and can act as a transcriptional repressor of methylated reporter genes [5]. Satellite cells, one of the first discovered adult stem cell populations, are responsible for postnatal muscle growth and regeneration following muscle damage. The majority of satellite cells remain quiescent in adult muscle but upon stimulation re-enter the cell cycle and proliferate as myoblasts to repopulate the satellite cell pool and undergo myogenic differentiation. Myogenin is activated early in this differentiation process and is one of the key transcription factors involved in coordinating myogenic differentiation. The Myogenin promoter in developing somites is initially methylated prior to activation and is subsequently demethylated [13]. The myoblast cell line $\mathrm{C} 2 \mathrm{C} 12$ can be induced to differentiate to myotubes upon serum starvation and faithfully recapitulates the activation of Myogenin during differentiation. Using a combination of these cells and in vivo muscle regeneration assays, Oikawa et al. show that expression of CIBZ and Myogenin proteins are reciprocally regulated when satellite cells are induced to differentiate: CIBZ levels are attenuated while Myogenin levels increase. Further investigation showed that changes in 
Myogenin levels are attributable to an upregulation of the mRNA, whereas changes in CIBZ levels appear to be at the protein level, suggesting that it is directly implicated in Myogenin regulation. Next, the authors show that the level of CIBZ protein is an important factor in myogenic differentiation, as manipulation of CIBZ levels in $\mathrm{C} 2 \mathrm{C} 12$ cells alters their propensity to undergo myogenesis in vitro. Specifically, reduction of CIBZ protein levels induced ectopic expression of Myogenin in $\mathrm{C} 2 \mathrm{C} 12$ cells and accelerated the formation of myotubes in vitro, whereas ectopically expressed CIBZ attenuated Myogenin upregulation upon differentiation. These reciprocal observations suggest that tight regulation of CIBZ levels are a key step in satellite cell differentiation. Importantly, the Myogenin gene appears to be a direct target of the CIBZ protein in a DNA methylation-dependent manner in vitro and in vivo, and is capable of repressing methylated Myogenin reporter constructs. Their data is in accordance with a model proposed by Joan Boyes and colleagues where DNA methylation helps to restrict Myogenin activation until both of its activation factors, MEF2A and SIX1, are co-expressed [13]. SIX1 is suggested to be required for demethylation and reorganization of chromatin at the promoter, leading to full expression. This model might also be applicable to the prevention of premature or inappropriate activation of a substantial number of other tissuespecific genes with an intermediate density of methylated CpGs at their promoters.

In summary, this work shows that CIBZ is required to suppress myogenic differentiation in satellite cells in vitro, most likely due to its role in the transcriptional repression of the key myogenic differentiation factor Myogenin. This provides support for the hypothesis that 'readers' are required to interpret the DNA methylation signal in order to exert its function on transcriptional repression (Figure 1). This study identifies an important selective target gene of methyl-CpG-binding proteins and suggests that they can play previously unappreciated and very specific roles in biological processes. As CIBZ expression is fairly ubiquitous, it will be interesting to uncover the wider role that this protein plays in gene repression, in addition to discovering more about its function in myogenesis, through constitutive and conditional mouse knockout studies. This study has also furthered our understanding of the molecular mechanisms that govern the maintenance and differentiation of an adult stem-cell population. In this respect it will be interesting to learn more about the regulation of this mechanism in growth and regeneration of skeletal muscle in vivo, in particular the dynamic
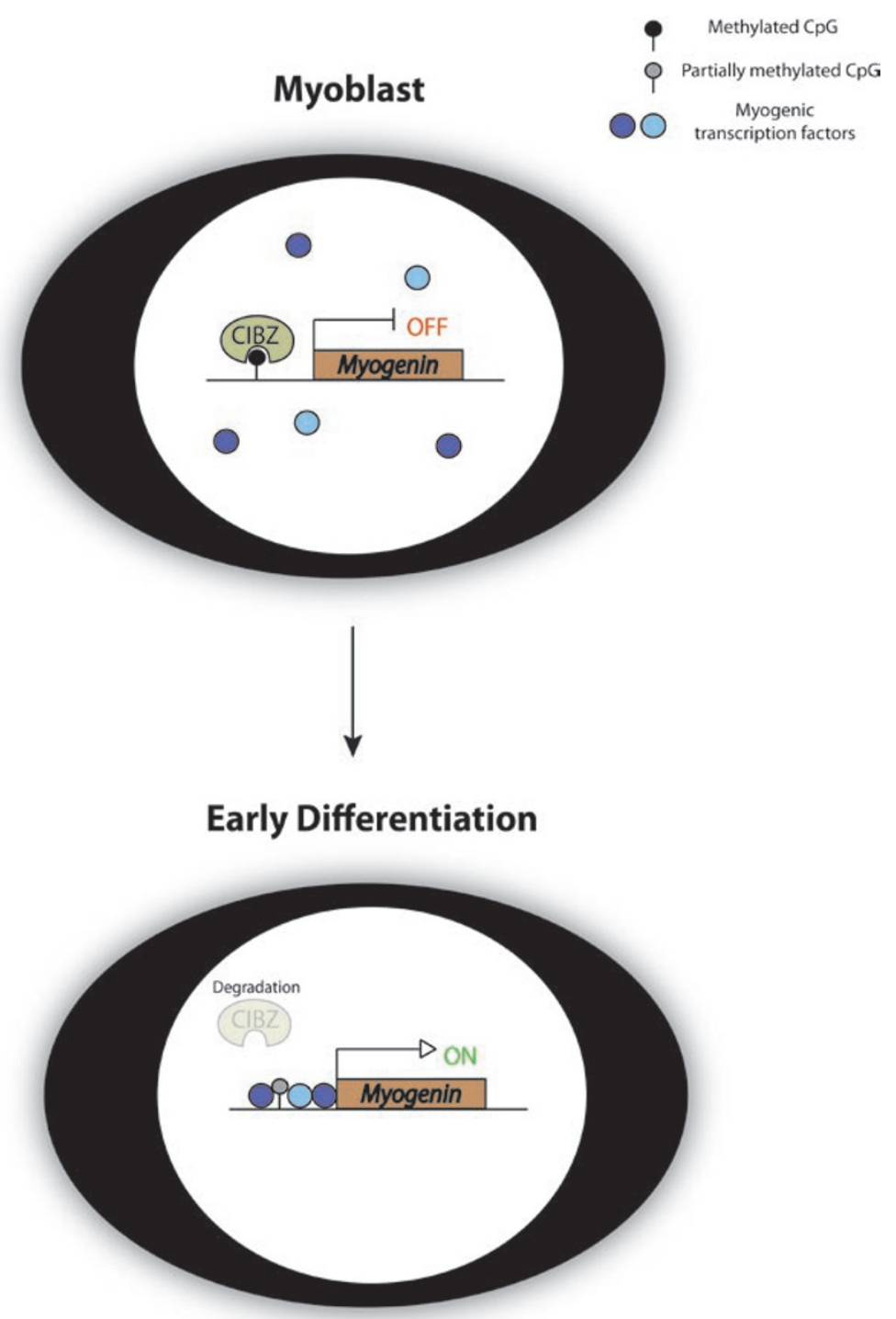

Figure $1 \mathrm{~A}$ model for Myogenin regulation during myogenesis. In undifferentiated myoblasts the Myogenin promoter is DNA methylated and bound by CIBZ. This negatively influences the binding of myogenic transcription factors to the promoter and maintains repression of Myogenin. Upon induction of differentiation the CIBZ protein is rapidly degraded and a reduction in DNA methylation at the promoter is observed, leading to increased binding of myogenic transcription factors and expression of Myogenin. For simplicity only one $\mathrm{CpG}$ is shown. 
regulation of the CIBZ protein during this process. Interestingly, in this regard, the ubiquitin-proteasome pathway has been linked to the rapid downregulation of CIBZ during myogenic differentiation [14]. Further investigation may reveal the regulatory mechanisms behind differentiation of satellite cells and in the future lead to a better understanding of myodegenerative diseases.

In light of previous studies of other methyl-CpG-binding proteins, the mutation of which cause only relatively mild phenotypes, it may have seemed a tall order to some that CIBZ would have a key role in transcriptional repression during a cell differentiation process. Coincidently, a single-nucleotide polymorphism in the human homolog of CIBZ, ZBTB38, was shown in a genome-wide association study to be the polymorphism most associated with human height [15]. The reason for this observation is unclear, but it is an interesting speculation that polymorphisms of ZBTB38 in a population may contribute to variation in human stature via a role in skeletal muscle differentiation and growth.

\section{References}

1 Taylor SM, Jones PA. Multiple new phenotypes induced in 10T1/2 and 3T3 cells treated with 5-azacytidine. Cell 1979; 17:771-779.

2 Lassar AB, Paterson BM, Weintraub H. Transfection of a DNA locus that mediates the conversion of $10 \mathrm{~T} 1 / 2$ fibroblasts to myoblasts. Cell 1986; 47:649656.

3 Davis RL, Weintraub H, Lassar AB. Expression of a single transfected cDNA converts fibroblasts to myoblasts. Cell 1987; 51:987-1000.

4 Sasai N, Defossez PA. Many paths to one goal? The proteins that recognize methylated DNA in eukaryotes. Int $J$ Dev Biol 2009; 53:323-334.

5 Filion GJ, Zhenilo S, Salozhin S, Yamada D, Prokhortchouk E, Defossez PA. A family of human zinc finger proteins that bind methylated DNA and repress transcription. Mol Cell Biol 2006; 26:169-181.

6 Prokhortchouk A, Sansom O, Selfridge $\mathrm{J}$, et al. Kaiso-deficient mice show resistance to intestinal cancer. Mol Cell Biol 2006; 26:199-208.

7 Li E, Bestor TH, Jaenisch R, et al. Targeted mutation of the DNA methyltransferase gene results in embryonic lethality. Cell 1992; 69:915-926.

8 Okano M, Bell DW, Haber DA, Li E. DNA methyltransferases Dnmt3a and Dnmt3b are essential for de novo methylation and mammalian development. Cell 1999; 99:247-257.

9 Stancheva I, Collins AL, Van den Veyver IB, Zoghbi H, Meehan RR. A mutant form of MeCP2 protein associated with human Rett syndrome cannot be displaced from methylated DNA by notch in Xenopus embryos. Mol Cell 2003; 12:425-435.

10 Ruzov A, Dunican DS, Prokhortchouk $\mathrm{A}$, et al. Kaiso is a genome-wide repressor of transcription that is essential for amphibian development. Development 2004; 131:6185-6194.

11 Oikawa Y, Omori R, Nishii T, Ishida Y, Kawaichi M, Matsuda E. The methylCpG-binding protein CIBZ suppresses myogenic differentiation by directly inhibiting myogenin expression. Cell Res 2011; 21:1578-1590.

12 Nabeshima Y, Hanaoka K, Hayasaka $\mathrm{M}$, et al. Myogenin gene disruption results in perinatal lethality because of severe muscle defect. Nature 1993; 364:532-535.

13 Palacios D, Summerbell D, Rigby PW, Boyes J. Interplay between DNA methylation and transcription factor availability: implications for developmental activation of the mouse Myogenin gene. Mol Cell Biol 2010; 30:3805-3815.

14 Oikawa Y, Matsuda E, Nishii T, Ishida Y, Kawaichi M. Down-regulation of CIBZ, a novel substrate of caspase-3, induces apoptosis. J Biol Chem 2008; 283:14242-14247.

15 Gudbjartsson DF, Walters GB, Thorleifsson $\mathrm{G}$, et al. Many sequence variants affecting diversity of adult human height. Nat Genet 2008; 40:609-615. 